\title{
Fever in the tropics: aetiology and case-fatality - a prospective observational study in a tertiary care hospital in South India
}

Siri Kratter Abrahamsen ${ }^{1,2+}$, Cathrine Nødtvedt Haugen ${ }^{1,2 \dagger}$, Priscilla Rupali ${ }^{3}$, Dilip Mathai ${ }^{3}$, Nina Langeland ${ }^{1,2}$, Geir Egil Eide 4,5 and Kristine Mørch ${ }^{1 *}$

\begin{abstract}
Background: The objective of this study was to describe aetiology and case fatality of fever among inpatients in a tertiary care hospital in South India.

Methods: This was an observational, prospective study conducted in a tertiary care hospital in Vellore, Tamil Nadu, India. Between July 2nd 2007 and August 2nd in 2007, adult patients admitted to the hospital with temperature $\geq$ $38.0^{\circ} \mathrm{C}$ were included consecutively and followed during the hospitalisation period. Demographic and clinical data were collected and analysed for each patient. Associations were sought between death and various clinical and demographic variables.

Results: One hundred patients were included, 61 male and 39 female. Mean age was 37.5 (range: 16 to 84) years. Mean fever duration was 5.4 (range: 0.1 to 42.9 ) weeks.

The following infectious aetiologies were recorded: tuberculosis (19\%), lower respiratory infection (11\%) including three with sepsis, urinary tract infection (10\%) including three with E. coli sepsis, Plasmodium falciparum malaria (5\%) including three patients with mixed P. vivax infection, scrub typhus (5\%), typhoid fever (4\%), cryptococcal meningitis (4\%) including three HIV positive patients, endocarditis (3\%) including two patients with Staphylococcus aureus sepsis, spleen abscess (2\%), amoebic liver abscess (2\%), sepsis undefined focus (1\%), HIV infection (1\%), hepatitis B (1\%), rubella (1\%), peritonitis (1\%) and cholecystitis (1\%).

Non-infectious causes of fever were diagnosed in 15\%, including systemic lupus erythematosus in four and malignancy in six patients. Cause of fever remained unknown in 13\%.

Case fatality during hospitalisation was 7\% (7/100). Six of those who died were male. Five fatalities had bacterial sepsis, one spleen abscess and malignancy, and one had lymphomalignant disorder.

Diabetes and increasing age were significant risk factors for fatal outcome in unadjusted analyses, but only increasing age was a risk factor for death in adjusted analysis.

Conclusions: A high number of tuberculosis and bacterial infections and a high case fatality rate from sepsis were found in this cohort, underlining the importance of microbiological diagnostics and targeted antimicrobial treatment in the management of fever. P. falciparum was identified in all malaria cases, and this rapidly fatal infection should be considered in patients with acute undifferentiated fever in India.
\end{abstract}

Keywords: Fever, Aetiology, Tropics, Case-fatality, Sepsis, Malaria, Tuberculosis

\footnotetext{
* Correspondence: kristine.morch@helse-bergen.no

${ }^{\dagger}$ Equal contributors

${ }^{1}$ Department of Medicine, National Centre for Tropical Infectious Diseases,

Haukeland University Hospital, Bergen, Norway

Full list of author information is available at the end of the article
} 


\section{Background}

Infectious diseases are leading causes of morbidity and death in tropical countries. The World Health Organization (WHO) reports that each of the main infectious aetiologies (that is, pneumonia, diarrhoea, HIV/AIDS, malaria, tuberculosis (TB) and neonatal infections), cause between 1.05 and 0.24 million deaths respectively per year in low income countries [1].

In resource-limited settings fever may be treated empirically or self-treated due to lack of access to diagnostic tests [2]. However, clinical algorithms in order to differentiate malaria, for instance, from other causes of fever are not specific [3-5]. Knowledge of local prevalence of infections is critical in order to target clinical work up and treatment.

Nevertheless, there are only a limited number of studies from India reporting on the aetiology of fever, and reliable surveillance data are not available [6]. The main objective of this study was to describe the aetiology of fever among patients in a tertiary care hospital in South India. The secondary objectives were: a) to describe to which extent laboratory and radiological tests were used as basis for aetiological diagnoses, and b) to report on the case fatality in this cohort and investigate associations between case fatality and various demographic and clinical variables.

\section{Methods}

\section{Study setting}

The study was conducted in a 1750-bed tertiary care referral hospital in Vellore, Tamil Nadu, South India.

\section{Design}

This was a prospective observational study among inpatients. Inclusion criteria were adult patients admitted to the hospital with fever of unlimited duration, with temperature $\geq 38.0^{\circ} \mathrm{C}$ upon admission. Co-morbidity was not an exclusion criterion. Patients were included consecutively during the four-week period from July 2nd 2007 to August 2nd 2007.

\section{Data collection and analysis}

Data were collected from hospital records, medical staff, and patients by a researcher not involved in patient care.

Demographic data, including age and gender, were recorded prospectively, as well as clinical data including fever duration, length of stay in hospital, human immunodeficiency virus (HIV) infection, antibiotic treatment before hospital admission, history of chronic disease, symptoms and findings, treatment, final diagnoses and deaths.

Radiological, biochemical and microbiological tests performed were registered. Tests had been ordered according to the discretion of the treating physicians, and no additional tests were conducted for the purposes of the study. Blood cultures were processed through automated "BacT Alert" Biomerieux inc. Malaria was diagnosed with microscopy slides stained with Leishman stain (Fisher scientific) and with quantitative buffy coat method (QBC) (QBC Diagnostics, USA). Further were the following microbiological tests used: Scrub typhus Detect IgM ELISA (InBios International, Inc, USA), Leptospira IgM ELISA (Panbio Pty., Ltd., Australia), Panbio Dengue Duo IgG/IgM Cassette (Inverness, Australia), Widal Ag kit (Febrile Antigen set, Span Diagnostics Ltd., Gujarat, India), Cryptococcus antigen Latex test (Remel Inc, USA), HIV Abbot Axsym Ag/Ab combo (Abbot, Germany), Genescreen HIV Ag/Ab (BIORAD, France), HIV TRIDOT (J Mitra, India), Abbot AxsymHBsAg ver 2 (Abbot, Germany), HBsAg EIA (Diasorin, Italy), Abbot Axsym - Anti HCV (Abbot, Germany), Ortho HCV 3.0 ELISA (Johnson and Johnson, USA).

When more than one diagnosis explaining fever had been noted in the records, the most probable aetiology (based on the available clinical information) was registered as the main aetiological diagnosis.

Data were recorded in a standardized clinical registration form.

For the patients who died, data were quality controlled from the hospital records retrospectively.

The association between death as a dependent variable and age, gender, fever duration, HIV infection and diabetes as explanatory variables were analysed by unadjusted and adjusted binary logistic regression analysis. The statistical analyses were performed using SPSS 18.0.

\section{Ethical considerations}

The study was approved by the Regional Ethics Committee of Norway and the Institutional Research Board at the study hospital in India.

\section{Results}

One hundred patients were included, 61 male and 39 female. Mean age was 37.4 years (range 16-84 years). Mean fever duration was 5.4 (range 0.1-42.9) weeks. Clinical characteristics are presented in Table 1.

Aetiological diagnoses, and associations with HIV infection and with sepsis, are shown in Table 2. Fifteen patients had more than one aetiological diagnosis.

Among patients diagnosed with TB $(\mathrm{n}=19)$ six patients had Mycobacterium tuberculosis detected in sputum samples, and for two of these it was a multi-drug resistant strain. Two patients had M. tuberculosis identified in lymph node biopsies. Two patients had findings consistent with $\mathrm{TB}$ on chest X-ray, and six on computer tomography $(\mathrm{CT})$. One TB patient was also diagnosed with hepatitis $\mathrm{C}$, and one with urinary tract infection (UTI). Seven patients were diagnosed with TB 
Table 1 Characteristics of 100 patients admitted with fever to a tertiary care hospital in South India during July 2007

\begin{tabular}{lr}
\hline Characteristics & Patients $(\mathbf{n}=\mathbf{1 0 0})$ \\
\hline Age (years) & \\
$\quad$ Mean (median, range) & $67.5(36.0,16-84)$ \\
Gender & 39 \\
$\quad$ Male (n) & \\
$\quad$ Female (n) & 34 \\
Fever duration (weeks) & 30 \\
Mean (median, range) & 26 \\
$<1$ week (n) & $9.4(2.1,0.1-42.9)$ \\
1-4 weeks (n) & \\
1-3 months (n) & \\
$>3$ months (n) & \\
Length of stay (days) & \\
Mean (median, range) & \\
Comorbidity & \\
HIV' (n) & \\
Diabetes (n) &
\end{tabular}

Missing values: Age, $\mathrm{n}=2$; Fever duration, $\mathrm{n}=2$.

Length of stay, $n=1$; Comorbidity, missing values interpreted as no comorbidity.

${ }^{1}$ Six were known HIV positive; among these one had a negative HIV test recorded, one was not tested, and four were HIV antibody positive.

meningitis, five on the basis of biochemical analyses of cerebrospinal fluid (CSF). All CSF cultures were TB negative. Two patients had findings consistent with meningeal involvement on CT scans.

Bacterial infections other than TB were diagnosed in 38 patients, and pathogens identified by culture are shown in Table 3.

Three out of 11 patients diagnosed with lower respiratory infection (LRI) died of sepsis. Diagnosis was confirmed in six patients by chest X-ray and in two by CT. One patient had Gram-negative rods in sputum, two patients had lung abscess.

Among 10 patients diagnosed with UTI, based on urine microscopy and culture, three had E. coli sepsis. Nosocomial UTI was found in 10 patients with other main causes of fever.

Plasmodium falciparum malaria was found in 5 patients based on Quantitative Buffy Coat (QBC) and thin and thick blood smears. Among these, three patients were diagnosed with mixed $P$. vivax infection. Parasitaemia in the range between $5 \%$ and $15 \%$ was recorded in three patients.

Scrub typhus $(\mathrm{n}=5)$ was diagnosed based on positive Weil Felix and positive IgM ELISA in one patient, while four were diagnosed based on symptoms and findings (headache, myalgia, lymphadenopathy and eschar). One of these patients also had positive HBsAg.
All patients diagnosed with cryptococcal meningitis $(\mathrm{n}=4)$ had cryptococcal Ag detected in CSF. One had disseminated infection with cryptococci isolated from blood, urine and CSF cultures. CT was negative in all four patients. Three of these patients had known untreated HIV, and among these one also had TB. One was HIV negative and had TB.

Typhoid fever $(\mathrm{n}=4)$ was diagnosed based on positive typhi-dot rapid test $(\mathrm{n}=3)$, Widal $(\mathrm{n}=2)$ and culture $(\mathrm{n}=4)$. All patients had Salmonella typhi $(\mathrm{n}=3)$ or Gram-negative rods $(\mathrm{n}=1)$ in blood culture. One patient had Gram-negative rods in blood culture and S. typhi in bone marrow biopsy.

Among patients with endocarditis $(\mathrm{n}=3)$, two had Staphylococcus aureus in blood culture and one of these patients died. One patient was culture negative. Two had rheumatic heart disease.

Spleen abscess $(n=2)$ was diagnosed based on clinical and radiological findings, and one of these died; one had Enterococcus spp. in blood culture.

Among patients diagnosed with sepsis $(n=9)$, five patients died. Microbiological and clinical information among these are shown in Tables 3 and 4 respectively.

Amoebic liver abscess $(n=2)$ was diagnosed based on clinical findings and abdominal ultrasound.

Five among seven patients with malignancy had lymphoid malignant disorder (LMD) based on biopsy from bone marrow, skin or lymph node, and among these one patient died who had spleen abscess as an infectious aetiological diagnosis of fever. One had adenocarcinoma in lung and brain, and one had leiomysarcoma. Systemic lupus erythematosus $(n=4)$ was diagnosed based on dsDNA, anti-nuclear antibodies and biochemical tests. Five patients had other non-infectious aetiological diagnoses as shown in Table 2. In $13 \%$ of patients a microbiological aetiology of fever was not identified; among these, those with fever duration less than three weeks were diagnosed as acute undifferentiated fever (AUF) and those with fever more than three weeks as pyrexia of unknown origin (PUO).

Diagnostic yields among radiological and microbiological tests performed in this cohort are shown in Table 5. Chest X-ray was performed in the majority of patients, and had a diagnostic yield of $8 \%$, while MRI was performed in 5 patients with a diagnostic yield of zero. HBsAg and hepatitis $\mathrm{C}$ antibodies were analysed in approximately two thirds of all patients, with a diagnostic yield of less than $3 \%$. Scrub typhus IgM ELISA was used as a diagnostic test in 20 patients; among these were all patients with AUF and PUO, with a diagnostic yield of $5 \%$. All positive malaria QBC tests $(n=5 / 77)$ were also analysed by microscopy, which then had a diagnostic yield of $100 \%$. Dengue IgM ELISA was used as a diagnostic test for four patients; one test result was 
Table 2 Aetiology of fever associated with HIV and with sepsis in a tertiary care hospital in South India during July $2007(n=100)$

\begin{tabular}{lccc}
\hline Aetiology of fever & Patients & Sepsis & HIV positive \\
& $n=100$ & $n=9$ & $n=6$ \\
\hline
\end{tabular}

\section{Bacteria}

Tuberculosis

TB meningitis $(n=7)$

TB other $(n=12)$

Lower respiratory infection

Urinary tract infection

Scrub typhus

Typhoid fever

Endocarditis

Sepsis other

Spleen abscess

Bacterial peritonitis

Cholecystitis

Parasites

Malaria

P. falciparum $(\mathrm{n}=2)$

$P$. falciparum $+P$. vivax $(\mathrm{n}=3)$

Amoebic liver abscess

\section{Fungi}

Cryptococcal meningitis

Crypt. meningitis and TB $(n=2)$

\section{Undefined}

AUF

PUO

Viral

HIV

Hepatitis B

Rubella

Malignancy

$\operatorname{LMD}(n=4)$

Pulmonary $(n=1)$

Leiomyosarcoma $(n=1)$

Non-infectious

SLE $(n=4)$

Toxic epidermal necrolysis $(n=1)$

Morbus Crohn $(n=1)$

Table 2 Aetiology of fever associated with HIV and with sepsis in a tertiary care hospital in South India during July $2007(n=100)$ (Continued)

SAPHO syndrome $(n=1)$

Arthritis $(n=1)$

Malignant neuroleptica syndrome

$(n=1)$

Data are presented as number of patients (n). One patient did not receive a final diagnosis.

Abbreviations: LMD lymphoid malignant disorder, TB tuberculosis, UTI urinary tract infection, HIV human immunodeficiency virus, AUF acute undifferentiated fever, PUO pyrexia of unknown origin, SLE systemic lupus erythematosus; SAPHO synovitis/acne/pustulosis/hyperossosis/osteitis.

'Escherichia coli septicaemia.

${ }^{2}$ Staphylococcus aureus septicaemia.

${ }^{3}$ Six were reported as HIV positive; among these one had a negative HIV test recorded, one was not tested, and four were HIV antibody positive.

positive but the patient was for unknown reasons not diagnosed with dengue fever.

Results were not available from $45 \%$ of chest $\mathrm{x}$-rays and $29 \%$ of blood cultures at the time of discharge. Serology results for scrub typhus and leptospirosis were not available for $45 \%$ and $40 \%$ of tests performed, respectively.

Case fatality in this study was 7\% (7/100). Characteristics, findings and treatment among patients who died in hospital are shown in Table 4. Two patients (with the diagnoses disseminated cryptococcosis and staphylococcal endocarditis) were discharged after only a brief hospital stay due to short expected lifespan, indicating that the case fatality rate of $7 \%$ is an underestimate in this cohort.

In unadjusted analysis, diabetes and increasing age were significant risk factors for a fatal outcome, while only age was found to be a risk factor for death in adjusted analysis (Table 6). Among men, 10\% died compared with 3\% of women, but this difference was not statistically significant. None of the HIV positive patients died during hospitalisation.

\section{Discussion}

In this study among a cohort of patients admitted to a tertiary care hospital in South India with fever, bacterial infections (38\%) and TB (19\%) were the most common aetiological diagnoses. Mean fever duration was high (5.4 weeks), and therefore the relatively high prevalence of TB is not surprising and is consistent with other hospital based studies reporting aetiology of prolonged fever in India. One study among 100 patients with fever of unknown origin (PUO) in Calcutta reported that TB (53\%), neoplasms (17\%) and collagen vascular disorders (11\%) were the dominant causes [7]. A similar pattern was found in two studies of PUO in North India, where infectious causes were diagnosed in approximately half of all cases, and $\mathrm{TB}$ was the most common infectious 
Table 3 Pathogens identified by culture among 100 patients admitted with fever to a tertiary care hospital in South India

\begin{tabular}{|c|c|c|c|c|c|c|}
\hline Pathogen & $\begin{array}{l}\text { Blood } \\
n=21\end{array}$ & $\begin{array}{l}\text { Urine } \\
n=24\end{array}$ & $\begin{array}{l}\text { Bone marrow } \\
\qquad n=1\end{array}$ & $\begin{array}{c}\text { Sputum } \\
n=13\end{array}$ & $\begin{array}{l}\text { CSF } \\
n=2\end{array}$ & $\begin{array}{c}\text { Lymph node } \\
\qquad \mathrm{n}=\mathbf{2}\end{array}$ \\
\hline \multicolumn{7}{|l|}{ Gram negative } \\
\hline Escherichia coli & $3^{1}(14.3)$ & $16(66.7)$ & & & & \\
\hline Klebsiella spp. & $1^{2}(4.8)$ & $1(4.2)$ & & & & \\
\hline S. typhi/paratyphi & $3(14.3)$ & & $1(100)$ & & & \\
\hline Pseudomonas spp. & & $1(4.2)$ & & $2(15.4)$ & & \\
\hline Acinetobact. anitratus & & & & $1(7.7)$ & & \\
\hline Gram negative rods & $1(4.8)$ & $1(4.2)$ & & $2(15.4)$ & & \\
\hline \multicolumn{7}{|l|}{ Gram positive } \\
\hline Staph. aureus & $2^{3}(9.5)$ & & & & & \\
\hline MRSA + Enterobact. & & & & $1(7.7)$ & & \\
\hline Enterococcus spp. & $1^{4}(4.8)$ & $4(16.7)$ & & & & \\
\hline Strept. viridans & $1^{5}(4.8)$ & - & & & & \\
\hline Gram positive cocci & & $1(4.2)$ & & & & \\
\hline Other & $8^{6}(38.1)$ & & & & & \\
\hline M. tuberculosis & & & & $6(46.2)$ & & $2(100)$ \\
\hline \multicolumn{7}{|l|}{ Fungi } \\
\hline Cryptococci & $1(4.8)$ & & & & $1(50.0)$ & \\
\hline Fungi not specified & & $2(8.3)$ & & $1^{2}(7.7)$ & $1^{2}(50.0)$ & \\
\hline
\end{tabular}

Data are given as number of positive samples with percentages in parentheses. Each patient may have had more than one positive sample.

Abbreviations: CSF cerebrospinal fluid, spp species, S. Salmonella, MRSA methicillin resistant Staphylococcus aureus, Strept. Streptococcus, Staph Staphylococcus, Enterobact. Enterobacteriaceae, M mycobacterium.

${ }^{1}$ Samples from three patients with urosepsis.

${ }^{2}$ Sepsis with undefined focus.

${ }^{3}$ Samples from two patients with endocarditis.

${ }^{4}$ Spleen abscess.

${ }^{5}$ Pulmonary infection and sepsis.

${ }^{6}$ Spores, 3; difteroides, 2; gram positive bacteria and difteroides, 1; micrococci, 1, staphylococcus spp, 1.

Table 4 Characteristics, diagnoses, microbiological findings and treatment among patients who died during hospitalisation in a cohort of 100 patients admitted with fever to a tertiary care hospital in South India $(\mathbf{n}=\mathbf{7})$

\begin{tabular}{|c|c|c|c|c|c|}
\hline $\mathbf{P}$ & Fever (days) & Final diagnosis & Microbiological findings & Anti-microbial treatment $^{1}$ & $\begin{array}{l}\text { LOS } \\
\text { (days) }\end{array}$ \\
\hline 1 & 105 & Spleen abscess & Blood culture: Diphteroides ${ }^{3}$ & Meropenem & 22 \\
\hline \multirow[t]{2}{*}{2} & 90 & $\mathrm{LMD}^{2}$ & Sputum: Acinetobacter anitratus & Metronidazol, ampicillin, cloxacillin, gentamycin & 20 \\
\hline & & & Urine: E-coli & & \\
\hline \multirow[t]{3}{*}{3} & 5 & Sepsis & Sputum: MRSA, enterobacteriaceae & Imipenem, penicillin, ciprofloxacin, metronidazol & 14 \\
\hline & & & Pseudomonas spp & & \\
\hline & & & Blood: Klebsiella & & \\
\hline 4 & 15 & Pulmonary infection and sepsis & None & Ampicillin & 3 \\
\hline 5 & 30 & Endocarditis and sepsis & Blood: Staphylococcus aureus & Penicillin G, cloxacillin & 1 \\
\hline 6 & 3 & Pulmonary infection and sepsis & Sputum and CSF: Fungus & Penicillin G & 16 \\
\hline 7 & 3 & Pulmonary infection and sepsis & Blood: Streptococcus viridans & Penicillin G, doxycyclin, cefotaxime & 7 \\
\hline
\end{tabular}

Abbreviations: $P$ patient, Neg. negative, LMD lymphomalignant disorder, UTI urinary tract infection, MRSA methicillin resistant Staphylococcus aureus, LOS length of stay, SLE systemic lupus erythematosus.

${ }^{1}$ Antibiotics given as combination treatment or successively.

${ }^{2}$ Hairy cell leukaemia found by bone marrow histology.

${ }^{3}$ Probably due to contamination of sample from skin. 
Table 5 Use of diagnostic procedures among 100 patients admitted with fever to a tertiary care hospital in South India

\begin{tabular}{|c|c|c|c|}
\hline \multirow[t]{2}{*}{ Diagnostic procedure } & \multirow{2}{*}{$\begin{array}{c}\text { Patients tested } \\
\text { n }\end{array}$} & \multicolumn{2}{|c|}{ Diagnostic yield $^{1}$} \\
\hline & & n & (\%) \\
\hline Chest $X$ ray & 92 & $13 / 166$ & $(7.8)^{2}$ \\
\hline$C T$ & 31 & $16 / 31$ & (51.6) \\
\hline MRI & 5 & $0 / 5$ & $(0.0)$ \\
\hline Abdominal US & 60 & $13 / 60$ & (21.6) \\
\hline HBsAg detection & 69 & $2 / 69$ & (2.9) \\
\hline Anti-HCV detection & 64 & $1 / 64$ & (1.6) \\
\hline Anti-HIV detection & 72 & $4 / 72$ & (5.6) \\
\hline Scrub typhus IgM ELISA & 20 & $1 / 20$ & $(5.0)^{3}$ \\
\hline Weil felix & 7 & $1 / 7$ & (14.3) \\
\hline Typhi dot & 36 & $3 / 36$ & (8.3) \\
\hline Widal & 14 & $3 / 14$ & $(21.4)$ \\
\hline Leptospira IgM ELISA & 15 & $0 / 15$ & $(0.0)^{4}$ \\
\hline Dengue IgM ELISA & 4 & $1 / 4$ & $(25.0)$ \\
\hline QBC & 77 & $5 / 134$ & (3.7) \\
\hline Malaria microscopy & 5 & $5 / 5$ & $(100)$ \\
\hline Stool microscopy & 17 & $1 / 17$ & (5.9) \\
\hline Blood culture, automated & 83 & $11 / 157$ & $(7.0)^{5}$ \\
\hline Urine culture & 42 & $21 / 42$ & $(50.0)$ \\
\hline CSF culture & 16 & $1 / 16$ & (6.3) \\
\hline Sputum culture & 53 & $10 / 53$ & (1.9) \\
\hline
\end{tabular}

Abbreviations: $C T$ computer tomography, MRI magnetic resonance imaging,

US ultrasound, HCV hepatitis C virus, HIV human immunodeficiency virus,

CSF cerebrospinal fluid, $H B S A g$ Hepatitis B surface antigen, $Q B C$ quantitative buffy coat.

${ }^{1}$ Positive tests among total number of tests performed.

${ }^{2} 74$ chest $X$-rays were not described at discharge.

${ }^{3}$ Results were not available for nine patients at discharge.

${ }^{4}$ Results were not available for six patients at discharge.

${ }^{5}$ Results from 46 blood cultures were not available at discharge.

aetiology [8,9]. In a study of PUO from the South of Turkey, the most important infectious causes (59\%) included TB (17\%), endocarditis (7\%), intra-abdominal abscess (7\%), brucellosis (6\%), and UTI (6\%) [10].

Bacterial infections and sepsis were associated with all the deaths recorded in this cohort, except for one patient who died due to malignancy. Not surprisingly among patients with severe bacterial infections, older age was a significant risk factor for death. In general, bacterial sepsis is probably undertreated in tropical countries for several reasons, including unspecific diagnosis of fever, high prevalence of multi-resistant microbes, lack of blood culture facilities, and widespread use of counterfeit drugs. This may explain the high case fatality reported in African patients with fever incorrectly treated as malaria [11]. In India, a retrospective study from Mumbai among 160 patients who died from acute febrile illness reported malaria in 23\%, leptospirosis in $22 \%$ and dengue fever in $2 \%$, while up to $54 \%$ died due to unexplained fever, a proportion of which is likely to have been caused by bacterial infections [12]. However, in our study, as many as 83 among 100 patients were tested with blood culture, and all patients with AUF and PUO had negative blood cultures (except one PUO who was not tested), suggesting that bacteraemia may not have been under-diagnosed in this cohort. Antimicrobial resistance patterns were not recorded in all patients in this study, but it is possible that antimicrobial resistance has had an impact on case fatality. Antimicrobial resistance is a well-described predictor of increased fatality rate in sepsis [13].

Malaria is known to be severely underreported and insufficiently controlled in India [6,14], and malaria overtreatment, at the cost of other potentially severe infections, has also been reported [15]. Malaria prevalence in South India has been reported to be low compared with states in North-East India [16]. Among hospitalised cases in Vellore, Tamil Nadu in 1998, a predominance of $P$. vivax infections $(\mathrm{n}=97)$ compared with $P$. falciparum $(\mathrm{n}=70)$ was reported [17]. In our study $5 \%$ of fever cases were due to malaria, all of which involved $P$. falciparum infections. A malaria prevalence of $17 \%$ among patients hospitalised with acute undifferentiated fever was reported from Vellore, India in 2010, and, among these, $57 \%$ were due to P. falciparum [18]. A similar increasing trend of $P$. falciparum predominance has been reported from Central India $[19,20]$.

The ratio of infectious causes of fever compared with non-infectious causes is usually higher among immunocompromised patients than immunocompetent individuals, as previously reported in two studies from hospitalized patients in India and Thailand [21,22]. In these studies, fever in HIV patients was caused by infections in $100 \%$ and $85 \%$ of patients respectively, with $\mathrm{TB}$ (69\% and 42\%), cryptococcal infections (10\% and $24 \%$ ) and Pneumocystis jiroveci pneumonia (7\% and 13\%) as the most common causes [21,22]. In our study, six patients had HIV infection, and three of these presented with cryptococcal infection.

Scrub typhus and dengue virus infection, which typically cause acute fever, were diagnosed in $5 \%$ and $0 \%$ of the patients respectively. These figures are low compared with $48 \%$ and $7 \%$, respectively, reported from a prospective study among patients from the same hospital in 2010 [18]. This difference may be explained by selection criteria or other study variables, including seasonal variations or the occurrence of epidemics. Our study was conducted during the hot dry season (July), while Chrispal et al. included patients for 12 consecutive months. Scrub typhus is more prevalent during cooler months, and outbreaks of dengue are reported in the 
Table 6 Logistic regression of deaths in a cohort of 100 patients admitted with fever in a tertiary hospital in South India associated with characteristics and comorbidity

\begin{tabular}{|c|c|c|c|c|c|c|c|c|c|c|c|c|}
\hline \multirow[t]{3}{*}{ Variables } & \multirow[t]{3}{*}{$\mathrm{N}$} & \multicolumn{11}{|c|}{ Deaths $(n=7)$} \\
\hline & & \multirow{2}{*}{$\begin{array}{c}\text { Yes } \\
\text { n (\%) }\end{array}$} & \multirow{2}{*}{$\begin{array}{c}\text { No } \\
\text { n (\%) }\end{array}$} & \multicolumn{3}{|c|}{ Unadjusted $^{\mathrm{a}}$} & \multicolumn{3}{|c|}{ Fully adjusted $^{\mathbf{b}}$} & \multicolumn{3}{|c|}{ Final model ${ }^{c}$} \\
\hline & & & & OR & $95 \% \mathrm{Cl}$ & p-value & OR & $95 \% \mathrm{Cl}$ & $\mathrm{p}$-value & OR & $95 \% \mathrm{Cl}$ & $\mathrm{p}$-value \\
\hline \multicolumn{13}{|l|}{ Gender } \\
\hline Women & 39 & $1(2.6)$ & 38(97.4) & Ref. & & & Ref. & & & & & \\
\hline Men & 60 & $6(10.0)$ & $54(90.0)$ & 4.22 & $(0.49,36.51)$ & 0.191 & 4.72 & $(0.44,51.16)$ & 0.202 & & & \\
\hline \multicolumn{13}{|l|}{ Age } \\
\hline per 10 years $^{\mathrm{d}}$ & 98 & 56.7 & 36.0 & 2.46 & $(1.38,4.37)$ & 0.002 & 2.42 & $(1.20,4.91)$ & 0.014 & 2.44 & $(1.37,4.34)$ & 0.002 \\
\hline Fever duration $^{d}$ & 98 & 5.4 & 5.1 & 1.00 & $(0.90,1.10)$ & 0.930 & 0.97 & $(0.83,1.12)$ & 0.692 & & & \\
\hline \multicolumn{13}{|l|}{ Comorbidity } \\
\hline Diabetes & 24 & $4(16.7)$ & 19(79.2) & 5.20 & $(1.07,25.20)$ & 0.041 & 1.15 & $(0.14,9.38)$ & 0.898 & & & \\
\hline HIV & 6 & $0(0.0)$ & $6(100)$ & .00 & 0.00 & 0.999 & 0.00 & .00 & 0.999 & & & \\
\hline
\end{tabular}

Abbreviations: $O R$ odds ratio, $\mathrm{Cl}$ confidence interval, $H I V$ human immune deficiency virus.

asimple binary logistic regression analyses

${ }^{\mathrm{b}}$ Multiple binary logistic regression analysis including all variables in one model.

${ }^{c}$ Backward stepwise elimination of variables with likelihood ratio test $p$-value $\leq 0.05$ starting from fully adjusted model.

${ }^{\mathrm{d} D a t a}$ are given as mean age (years) and mean fever duration (weeks).

area [23-26], but the difference may also be due to undetected cases. In our study cohort, only four cases were tested for dengue infection, and only nine out of 20 results of tests for Scrub typhus were available at the time of discharge.

The prevalence of leptospirosis in the area is known to be high; Chrispal et al. detected 12 cases of leptospirosis among hospitalised cases of fever (3\%) in 2010, and a seroprevalence of approximately $70 \%$ has been reported in exposed risk groups, such as rice mill farmers, in the area [27]. Leptospirosis may potentially have been under diagnosed in our cohort, since only nine serological tests were available at the time of discharge.Hantavirus infection in humans in India was first reported from Vellore [28], and is most probably under diagnosed in India, as reported by Chandy et al. [29]. Hantavirus was found in one case among 398 hospitalised AUF patients in Vellore by Chrispal et al. [18]. Hantavirus serology was not performed in patients in our cohort. Furthermore, Brucella serology was not performed, and since blood cultures need three weeks incubation to detect Brucella spp., this is another example of an infection that potentially may have been overlooked.

The aetiology of fever among travellers to the tropics differs from that among residents due to pattern of exposure and immunity, as was shown in a study among 1743 western travellers with fever imported from the tropics in Belgium, where TB was diagnosed in only $2 \%$ [30]. In this large study, travellers to Asia were diagnosed with LRI (13\%), dengue fever (12\%), non-falciparum malaria (9\%), bacterial enteritis (9\%), mononucleosis-like syndrome (7\%), skin/soft tissue infection (4\%), enteric fever (3\%), genitourinary infection (3\%) and P. falciparum malaria (2\%), while $19 \%$ had fever of unknown aetiology [30]. A study among travellers from Asia in Australia reported a similar pattern with bacterial pneumonia, dengue fever, malaria, hepatitis A and typhoid fever reported as relatively common infections, while TB was diagnosed in only $0.4 \%$ [31].

The pattern of fever aetiology found in this study would not be representative for the causes of fever in the general population. There are three main limitations explaining this. The selection of patients admitted to a tertiary care hospital in India is biased as a result of factors such as severity of disease, gender and accessibility. In this study only adults where included, while children would expect to have different fever aetiologies due to exposure and immunity. Entomological factors cause seasonal and geographical variations in vector borne diseases in India, and our study selecting patients during a dry month of the year in South India would not incorporate such variations.

Further studies are needed, both population- and hospital based, in order to provide more evidence based information about the prevalence of infectious diseases in India.

\section{Conclusion}

A high number of bacterial and TB infections were found in a cohort of adult patients admitted to a tertiary care hospital with fever in South India, and there was a relatively high case fatality rate from sepsis. This underlines the importance of establishment of relevant microbiological diagnostics and targeted antimicrobial treatment in the management of fever. P. falciparum was identified in all malaria cases, and this rapidly fatal 
infection should be considered in all patients with acute undifferentiated fever in India.

\section{Consent}

Written informed consent was obtained from the patients for the publication of this report.

\section{Competing interests}

The authors declare that they have no competing interests.

\section{Authors' contributions}

SKA, CNH, KM, NL and DM designed the study. Collection of prospective data was performed by SKA and CNH, supervised by DM and PR. Statistical analyses were performed by GEE and KM. All authors contributed to interpretation of results and revision of the manuscript. All authors read and approved the final manuscript.

\section{Author details}

'Department of Medicine, National Centre for Tropical Infectious Diseases, Haukeland University Hospital, Bergen, Norway. ${ }^{2}$ Institute of Medicine, University of Bergen, Bergen, Norway. ${ }^{3}$ Department of Medicine Unit 1 , Christian Medical College, Vellore, Tamil Nadu, India. ${ }^{4}$ Centre for Clinical Research, Haukeland University Hospital, Bergen, Norway. ${ }^{5}$ Department of Global Public Health and Primary Care, University of Bergen, Bergen, Norway.

Received: 28 June 2012 Accepted: 17 July 2013

Published: 30 July 2013

\section{References}

1. WHO: The 10 leading causes of death by broad income group (2008); 2011

2. Chaturvedi HK, Mahanta J, Pandey A: Treatment-seeking for febrile illness in north-east India: an epidemiological study in the malaria endemic zone. Malar J 2009, 8(1):301.

3. Chandramohan D, Jaffar S, Greenwood B: Use of clinical algorithms for diagnosing malaria. Trop Med Int Health 2002, 7(1):45-52.

4. Chandramohan D, Carneiro I, Kavishwar A, Brugha R, Desai V, Greenwood B: A clinical algorithm for the diagnosis of malaria: results of an evaluation in an area of low endemicity. Trop Med Int Health 2001, 6(7):505-510.

5. Mwangi TW, Mohammed M, Dayo H, Snow RW, Marsh K: Clinical algorithms for malaria diagnosis lack utility among people of different age groups. Trop Med Int Health 2005, 10(6):530-536.

6. John TJ, Dandona L, Sharma VP, Kakkar M: Continuing challenge of infectious diseases in India. Lancet 2011, 377(9761):252-269.

7. Kejariwal D, Sarkar N, Chakraborti SK, Agarwal V, Roy S: Pyrexia of unknown origin: a prospective study of 100 cases. J Postgrad Med 2001, 47(2):104-107.

8. Handa R, Singh S, Singh N, Wali JP: Fever of unknown origin: a prospective study. Trop Doct 1996, 26(4):169-170.

9. Sharma BK, Kumari S, Varma SC, Sagar S, Singh S: Prolonged undiagnosed fever in northern India. Trop Geogr Med 1992, 44(1-2):32-36.

10. Saltoglu N, Tasova Y, Midikli D, Aksu HS, Sanli A, Dundar IH: Fever of unknown origin in Turkey: evaluation of 87 cases during a nine-year -period of study. J Infect 2004, 48(1):81-85.

11. Reyburn H, Mbatia R, Drakeley C, Carneiro I, Mwakasungula E, Mwerinde O, Saganda K, Shao J, Kitua A, Olomi R, et al: Overdiagnosis of malaria in patients with severe febrile illness in Tanzania: a prospective study. BMJ 2004, 329(7476):1212.

12. Bajpai LB: Mortality analysis of patients of acute febrile illness during monsoon in a tertiary care hospital of Mumbai. Infect Dis Clin Pract 2008, 16(5):294-297.

13. Blomberg B, Manji KP, Urassa WK, Tamim BS, Mwakagile DS, Jureen R, Msangi V, Tellevik MG, Holberg-Petersen M, Harthug S, et al: Antimicrobial resistance predicts death in Tanzanian children with bloodstream infections: a prospective cohort study. BMC Infect Dis 2007, 7:43.

14. Dhingra N, Jha P, Sharma VP, Cohen AA, Jotkar RM, Rodriquez PS, Bassani DG, Suraweera W, Laxminarayan R, Peto R: Adult and child malaria mortality in India: a nationally representative mortality survey. Lancet 2010, 376(9754):1768-1774.

15. Joshi R, Colford JM Jr, Reingold AL, Kalantri S: Nonmalarial acute undifferentiated fever in a rural hospital in central India: diagnostic uncertainty and overtreatment with antimalarial agents. Am J Trop Med Hyg 2008, 78(3):393-399.

16. Kumar A, Valecha N, Jain T, Dash AP: Burden of malaria in India: retrospective and prospective view. Am J Trop Med Hyg 2007, 77(6 Suppl):69-78.

17. Harris VK, Richard VS, Mathai E, Sitaram U, Kumar KV, Cherian AM, Amelia SM, Anand G: A study on clinical profile of falciparum malaria in a tertiary care hospital in south India. Indian J Malariol 2001, 38(1-2):19-24

18. Chrispal A, Boorugu H, Gopinath KG, Chandy S, Prakash JA, Thomas EM, Abraham AM, Abraham OC, Thomas K: Acute undifferentiated febrile illness in adult hospitalized patients: the disease spectrum and diagnostic predictors - an experience from a tertiary care hospital in South India. Trop Doct 2010, 40(4):230-234.

19. Singh N, Kataria O, Singh MP: The changing dynamics of Plasmodium vivax and P. falciparum in central India: trends over a 27-year period (1975-2002). Vector Borne Zoonotic Dis 2004, 4(3):239-248.

20. Singh N, Chand SK, Mishra AK, Bharti PK, Singh MP, Ahluwalia TP, Dash AP: Epidemiology of malaria in an area of low transmission in central India. Am J Trop Med Hyg 2006, 75(5):812-816.

21. Rupali P, Abraham OC, Zachariah A, Subramanian S, Mathai D: Aetiology of prolonged fever in antiretroviral-naive human immunodeficiency virusinfected adults. Nat/ Med J India 2003, 16(4):193-199.

22. Kitkungvan $D$, Apisarnthanarak $A$, Plengpart $P$, Mundy LM: Fever of unknown origin in patients with HIV infection in Thailand: an observational study and review of the literature. Int J STD AIDS 2008, 19(4):232-235.

23. Samuel PP, Thenmozhi $V$, Tyagi BK: A focal outbreak of dengue fever in a rural area of Tamil Nadu. Indian J Med Res 2007, 125(2):179-181.

24. Paramasivan R, Thenmozhi V, Kabilan L, Tewari SC, Arunachalam N, Dhananjeyan KJ, Tyagi BK: Seroepidemiology of a focal outbreak of dengue in Tamil Nadu. Indian J Med Res 2006, 124(6):718-720.

25. Kamarasu K, Malathi M, Rajagopal V, Subramani K, Jagadeeshramasamy D, Mathai E: Serological evidence for wide distribution of spotted fevers \& typhus fever in Tamil Nadu. Indian J Med Res 2007, 126(2):128-130.

26. Mathai E, Rolain JM, Verghese GM, Abraham OC, Mathai D, Mathai M, Raoult D: Outbreak of scrub typhus in southern India during the cooler months. Ann N Y Acad Sci 2003, 990:359-364.

27. Natarajaseenivasan K, Boopalan M, Selvanayaki K, Suresh SR, Ratnam S: Leptospirosis among rice mill workers of Salem South India. Jpn J Infect Dis 2002, 55(5):170-173.

28. Chandy S, Yoshimatsu K, Ulrich RG, Mertens M, Okumura M, Rajendran P, John GT, Balraj V, Muliyil J, Mammen J, et al: Seroepidemiological study on hantavirus infections in India. Trans $R$ Soc Trop Med Hyg 2008, 102(1):70-74.

29. Chandy S, Abraham S, Sridharan G: Hantaviruses: an emerging public health threat in India? A review. J Biosci 2008, 33(4):495-504.

30. Bottieau E, Clerinx J, Van den Enden E, Van Esbroeck M, Colebunders R, Van Gompel A, Van den Ende J: Fever after a stay in the tropics: diagnostic predictors of the leading tropical conditions. Medicine (Baltimore) 2007, 86(1):18-25.

31. O'Brien D, Tobin S, Brown GV, Torresi J: Fever in returned travelers: review of hospital admissions for a 3-year period. Clin Infect Dis 2001, 33(5):603-609.

doi:10.1186/1471-2334-13-355

Cite this article as: Abrahamsen et al:: Fever in the tropics: aetiology and case-fatality - a prospective observational study in a tertiary care hospital in South India. BMC Infectious Diseases 2013 13:355. 